\title{
COMO O DISTRITO FEDERAL CUIDA DA SAÚDE DE SEUS IDOSOS
}

\section{HOW THE FEDERAL DISTRICT TAKES CARE OF THE HEALTH OF THEIR ELDERLY}

\section{${ }^{1,2}$ Maria da Conceição Sousa e Silva; ${ }^{3}$ Danielle Silva Araújo; ${ }^{4}$ Paulo Henrique Rosa Martins}

\section{RESUMO}

Introdução: $\mathrm{O}$ envelhecimento populacional é um fenômeno que ocorre em todas as regiões do mundo, e este processo gera mudanças na estrutura etária de um povo resultando em maior número de pessoas idosas. O estudo descreve sobre o envelhecimento populacional saudável e como o Distrito Federal cuida de sua população idosa. Objetivo: destacar o papel do Núcleo de Saúde do Idoso na promoção da qualidade de vida e saúde destes no Distrito Federal. Metodologia: foi realizada uma pesquisa desenvolvida mediante revisão bibliográfica, com levantamento e análise das publicações de 1992 a 2014, com enfoque no tema proposto. Foram utilizados como base de dados SciELO, LILACS e BIREME. Resultados e discussão: Observou-se a necessidade de maior divulgação das práticas integrativas de saúde desenvolvidas nas Unidades Básicas de Saúde (UBS), visando construir na comunidade em questão um estilo de vida ativo para a manutenção da autonomia física e mental no processo para um envelhecimento saudável. Conclusão: O estudo evidenciou o aumento da população com 60 anos ou mais no Brasil e em Brasília. Ressaltando por dados estatísticos que Brasília apresenta características econômicas e sociais que a diferencia de outras unidades da federação.

Palavras-chave: Envelhecimento populacional. Envelhecimento saudável. Saúde do idoso. Politica Distrital de Idoso.

\footnotetext{
${ }^{1}$ Graduada em Enfermagem, Faculdade JK, Brasília-Distrito Federal, Brasília.

${ }^{2}$ Autor correspondente: figueira rodrigues@yahoo.com.br

${ }^{3}$ Patologia Molecular, Faculdade de Medicina, Universidade de Brasília (UnB), Brasília - Distrito Federal, Brasil.

${ }^{4}$ Biologia Microbiana, Instituto de Biologia, Universidade de Brasília (UnB), Brasília - Distrito Federal, Brasil. Data do recebimento: 10/02/2016

Data da aprovação: 25/03/2016
} 


\begin{abstract}
Introduction: Population aging is a phenomenon that occurs in all regions of the world, and this process generates changes in the age structure of a people resulting in people that are more elderly. The study describes about healthy aging population and as the Federal District takes care of its elderly population. Objective: highlight the role of the Senior Health Center in promoting quality of life and health of those in the Federal District. Methodology: was carried out a survey developed by literature review, a survey and analysis of 1992-2014 publications, focusing on the theme. It was used as database SciELO, LILACS and BIREME. Results and discussion: there was the need for greater disclosure of integrative health practices developed in Basic Health Units (BHU), to build in that community an active lifestyle to maintain physical and mental autonomy in the process of healthy aging. Conclusion: The study showed the increase in the population aged 60 or more in Brazil and Brasilia. Underscoring by statistical data that Brasilia has economic and social characteristics that differentiates it from other federation units.
\end{abstract}

Keywords: Population ageing. Healthy aging. Healthy aging. District Elderly Policy.

\title{
INTRODUÇÃO
}

O processo de envelhecimento gera mudanças na estrutura etária de um povo, resultando em um maior número de idosos em relação ao total da população, ocasionando mudanças no perfil demográfico e epidemiológico do país. Esta mudança é resultado da diminuição da fecundidade e mortalidade. Sendo a baixa taxa de fecundidade um processo influenciado pelos novos padrões familiares, acesso aos contraceptivos, maior participação da mulher no mercado de trabalho e maior grau de instrução (KANSO, 2013; CARNEIRO et al., 2013). Assim também ocorre com a queda na taxa de mortalidade, justificada por fatores como o avanço da medicina, acesso a saúde pública, alimentação, melhores condições sanitárias, ambientais e de moradia. Tais comportamentos são apontados como consequência para o aumento da longevidade (CARNEIRO et al., 2013).

Segundo dados das Nações Unidas, no ano de 2011 cerca de $11 \%$ da população era formada por idosos e estima-se que para o ano de 2050 esse percentual aumente para $22 \%$, chegando a aproximadamente 2 bilhões de idosos. (KANSO, 2013).

No Brasil, a transição demográfica do ponto de vista do gênero destaca a feminilização da velhice, onde a mulher representa $55,5 \%$ da população idosa, condição atribuída a maior expectativa de vida da mulher, que em média vive 8 anos a mais que os homens, fato justificado pela maior assiduidade da mulher aos programas de saúde (KUCHEMANN, 2012). 
A população idosa brasileira dobrou nos últimos vinte anos, vários fatores contribuíram para esse evento como, expectativa de vida aumentada e consequentemente a longevidade, condição que pode ser alcançada quando é possível ter uma boa qualidade de vida, baseada em um envelhecimento com autonomia, independência, boa saúde física, mental e social (BRASIL, 2013; VILELA et al., 2006).

O Distrito Federal (DF) reproduz o fenômeno que vem ocorrendo no Brasil como um todo, embora em ritmo menos acentuado. Isto pode ser observado no trabalho realizado por Lima (2011), onde foi possível constatar o aumento da população idosa de 1,2\% no ano de 1960 para 5,3\% no ano de 2000, caracterizando o envelhecimento populacional gradativo.

As características específicas da capital da república, onde possui os melhores indicadores sociais e de renda média elevada, os idosos representam um segmento com participação efetiva na renda local e no universo eleitoral, condição que levou na esfera governamental a formulação e implementação de políticas públicas voltadas ao idoso, culminando no DF e nas outras unidades da federação a criação das secretarias de estado específicas para o segmento (MIRAGAYA et al., 2013).

Tal situação coloca o DF com uma expectativa de vida considerada a mais alta do país, com possibilidade de viver até 75,3 anos, sendo que as mulheres podem alcançar os 79,2 anos de idade. Este segmento repete o modelo brasileiro, vivendo em núcleos familiares, partilhando da companhia do cônjuge, filhos e muitas vezes netos, sendo em grande parte responsável pelos cuidados destes (LIMA, 2011).

A secretaria de estado de saúde do Distrito Federal no uso de suas atribuições criou o Núcleo de Saúde do Idoso (NUSI) implantado em 1992, objetivando dar ênfase na promoção da saúde, prevenção, reabilitação e tratamento de agravos, onde seu universo é a população com idade igual ou superior a 60 anos. O Núcleo desenvolve suas atividades de modo descentralizado, visando a autonomia das regionais de saúde, ofertando suporte técnicocientifico para supervisão, avaliação e capacitação de recursos humanos, além de promover a organização de serviços, levantamento de dados epidemiológicos, elaboração de material educativo e informativo (SES-DF,1992).

Dentre os materiais elaborados pelo NUSI o Protocolo de Atenção à Saúde do Idoso do Distrito Federal usa o modelo de atenção primária a saúde, na intenção de promover melhorias na qualidade de vida, por meio de um acompanhamento sistemático nas Unidades Básicas de Saúde (UBS), para que seja possível reconhecer riscos de vulnerabilidades e ou fragilidades, para que possa intervir precocemente visando a autonomia e a independência nessa população, pois são condições indispensáveis para uma boa qualidade de vida na velhice (SES - DF,2014). 
O protocolo teve como documentos norteadores: a Política Distrital do Idoso - (Lei $\mathrm{n}^{\mathrm{o}} 3.822$, de 08 de fevereiro de 2006), a Política Nacional de Saúde da Pessoa Idosa (Portaria nº 2528, de 19 de outubro de 2006), Política Nacional do Idoso (Lei n ${ }^{\circ} 8842$, de 04 de janeiro de 1994) e o Estatuto do Idoso (Lei n ${ }^{\circ} 10.741$, de 01 de outubro de 2003) (BRASIL, 2014).

O Núcleo de Saúde do Idoso (NUSI) é um núcleo dentro da administração central da Secretaria de Estado de Saúde (SES), subordinado à Gerência de Ciclos de Vida (GCV), Diretoria do ciclo de vida e Práticas Integrativas à saúde (DCVPIS) e a Subsecretaria de Atenção Primária de Saúde (SAPS), ele é composto por 3 servidores, sendo uma terapeuta ocupacional, uma enfermeira, uma médica geriatra. O atendimento ao idoso é realizado em todas as Unidades Básicas de Saúde (Centros de Saúde, Clinicas da Família, Equipe de Saúde da Família, Postos de Saúde Urbanos e Rurais), pois a atenção primária é a porta principal para o acesso à rede de saúde do DF (SES-DF, 1992).

$\mathrm{O}$ acolhimento aos idosos deve ser feito por qualquer servidor da unidade, embora algumas ações sejam realizadas preferencialmente pelo enfermeiro; como consulta de enfermagem com avaliação multidimensional do idoso, abordagem familiar, assim como reconhecer na área de abrangência rede de apoio para encaminhamento das demandas que surgirem (SES-DF, 2014).

Estudos sobre a qualidade de vida da população idosa do DF, indicam que a promoção da saúde está além do fato da pessoa não se apresentar doente, mas sim relacionada também a fatores como ter mobilidade e boas relações sociais, isto resulta em uma boa qualidade de vida para este indivíduo (STIVAL et al., 2014).

Entendendo que a melhoria na qualidade de vida da pessoa idosa passa por uma assistência direcionada, a SES-DF por meio da Gerência de Práticas Integrativas de Saúde (GERPIS) desenvolveu a produção de conhecimento na área das Práticas Integrativas em Saúde (PIS) no âmbito do Sistema Único de Saúde (SUS - DF) visando promoção, prevenção, proteção, recuperação e reabilitação da saúde de modo integral e humanizada (SES-DF,2014).

As práticas integrativas em saúde mostram o exemplo de políticas públicas de caráter emancipatório, que promovem o protagonismo, a corresponsabilidade, autonomia e autodeterminação do indivíduo objetivando promover, manter e recuperar a saúde. Dentre as PIS desenvolvidas no DF, podemos destacar: Acupuntura, Arteterapia, Automassagem, Fitoterapia, Hatha Yoga, Homeopatia, Lian Gog, Madicina e Terapias Antroposóficas, Meditação, Musicoterapia, Reiki, Shantala, Tai Chi Chuan e Terapia Comunitária Integrativa (GERPIS, 2011). 
Sendo assim o presente trabalho teve como objetivo central descrever de que forma o Distrito Federal se articula para promover a saúde e o bem-estar de sua população idosa.

\section{METODOLOGIA}

O método utilizado foi a revisão bibliográfica, com levantamento e análise das publicações com enfoque em responder a seguinte questão norteadora: como o Distrito Federal promove a qualidade de vida dos seus idosos. O levantamento bibliográfico realizou-se no período de março a junho de 2015, mediante consulta em base de dados da literatura latinoamericana indexados nos bancos de dados SciELO (Scientific Eletronic Library Online), LILACS (Literatura Latino - Americana em Ciências da Saúde) e BIREME (Centro LatinoAmericano e do Caribe de Informação em Ciências da Saúde), utilizando como descritores: envelhecimento populacional, envelhecimento saudável, saúde do idoso, política distrital do Idoso.

Foram selecionados 10 artigos, publicados entre os anos de 1992 a 2014. Além disto, foram utilizados dados fornecidos pela Secretaria de Estado de Saúde do Distrito Federal e documentos disponíveis na página do Programa de Saúde do Idoso (Politica distrital do idoso, Política Nacional de Saúde da Pessoa Idosa, Política Nacional do Idoso e Estatuto do Idoso). Optou-se por utilizar artigos disponíveis na integra, na língua portuguesa e que atenderam à questão norteadora. Foram excluídos os artigos por não contemplarem o tema proposto pela pesquisa de modo objetivo. Após seleção dos artigos realizou-se leitura interpretativa e crítica necessária à elaboração do trabalho.

\section{RESULTADOS E DISCUSSÃO}

Atualmente o Núcleo de Saúde do Idoso (NUSI) atende cerca de 256.775 idosos distribuídos entre todas as regionais de saúde do DF conforme Tabela 1. 
Tabela 1: Resumo da pulação de Idosos de DF 2014

\begin{tabular}{|c|c|c|c|}
\hline Regional de Saúde & Pop. Total & Idosos & $\%$ de idosos \\
\hline Regional Norte & 278.397 & 34.494 & 12,4 \\
\hline Regional Sul & 130.700 & 27.735 & 21,2 \\
\hline Brazlândia & 63.531 & 5.249 & 8,3 \\
\hline CNBPRF & 146.020 & 12.167 & 8,3 \\
\hline Ceilândia & 445.058 & 37.860 & 8,5 \\
\hline Gama & 149.158 & 16.767 & 11,2 \\
\hline Paranoá & 132.275 & 7.338 & 5,5 \\
\hline Planaltina & 188.990 & 13.096 & 6,9 \\
\hline Recanto das Emas & 137.479 & 6.377 & 4,6 \\
\hline Samambaia & 219.694 & 12.448 & 5,7 \\
\hline Santa Maria & 130.076 & 7.083 & 5,4 \\
\hline São Sebastião & 93.318 & 3.343 & 3,6 \\
\hline Guará & 155.624 & 17.333 & 11,1 \\
\hline Sobradinho & 176.067 & 15.572 & 8,8 \\
\hline Taguatinga & 405.975 & 39.912 & 9,8 \\
\hline DF & 2.852.372 & 256.775 & 9,0 \\
\hline
\end{tabular}

Fonte: Gerência de Informação e Análise de Situação em Saúde/DIVEP/SVS/SES, 2014

Stival e colaboradores, (2014) analisaram 277 idosos que frequentavam a Unidade Básica de Saúde da cidade de Ceilândia-DF, e observaram que eram aposentados, casados, com idade entre 60 a 65 anos, renda de até um salário mínimo, não moravam sozinhos e que a maioria era do sexo feminino. Além disto, apresentavam índice elevado de boa qualidade de vida justificado por terem boas relações sociais e ambientais, visto que relataram sentirem-se seguros em seu ambiente do lar, terem oportunidade de lazer e cuidados com a saúde. Os mesmos apontaram participação ativa em atividades de lazer, grupos de convivência e atividades na igreja, possibilitando assim maior convivência social. 
Drummond e Alves (2013), realizaram um trabalho com 93 idosos atendidos pela Estratégia de Saúde da Família no Paranoá-DF, com o objetivo de estabelecer o perfil socioeconômico, demográfico e funcional dessa comunidade. Após coleta e análise dos dados, constataram que o perfil dos idosos se destacou com predomínio de mulheres, casados (as), que moram acompanhados, com moradia própria, com renda de até um salário mínimo, com formação educacional até o ensino fundamental e faixa etária dos 60 a 69 anos. Fato também demonstrado em outros estudos.

Tanto Drummond e Alves, (2013) quanto Stival et al., (2014) tiveram como destaque a hipertensão arterial sistêmica (HAS), Diabetes Mellitus e histórico de quedas, como as principais morbidades apresentadas, fato este que pode representar futura complicação funcional.

As Práticas Integrativas de Saúde (PIS) foram criadas justamente com o objetivo de modificação e melhoria dos dados acima apresentados. Através da inserção dos idosos nesse programa, o DF visa não deixar que esses agravos se estabeleçam. Ao todo o DF conta com 14 PIS (Tabela 2), sendo que cada UBS realiza pelo menos uma dessas atividades.

\section{Tabela 2: Práticas Integrativas de Saúde-DF}

\begin{tabular}{l|l}
\hline \multicolumn{1}{c|}{ PIS } & \multicolumn{1}{c}{ OBJETIVOS } \\
\hline Acupuntura & $\begin{array}{l}\text { Controle da dor de diversas origens, normalização das funções } \\
\text { orgânicas autonômicas, sensoriais e motoras. }\end{array}$ \\
\hline Arte terapia & $\begin{array}{l}\text { Terapia individual ou em grupo que utiliza arte como desenho, } \\
\text { pintura, colagem, musica visando estimular o autoconhecimento. }\end{array}$ \\
\hline Automassagem & $\begin{array}{l}\text { É vista como importante meio de prevenção de doenças, além de } \\
\text { proporcionar visão integral do ser humano e de sua interação com a } \\
\text { natureza. }\end{array}$ \\
\hline Fitoterapia & $\begin{array}{l}\text { Tratamento com utilização de ervas medicinais em diferentes formas } \\
\text { farmacêutica com finalidade profilática, curativa ou paliativa. }\end{array}$ \\
\hline Hatha yoga & $\begin{array}{l}\text { Promove redução do estresse, equilíbrio do sono, aumenta a } \\
\text { capacidade de concentração e criatividade, reeducação mental, } \\
\text { melhora o humor, favorecendo a qualidade de vida dos praticantes. }\end{array}$ \\
\hline Homeopatia & $\begin{array}{l}\text { Terapêutica que utiliza medicamentos de origem animal, vegetal e } \\
\text { mineral, em doses diluídas e dinamizadas, que estimula os } \\
\text { mecanismos naturais de cura do organismo. }\end{array}$ \\
\hline
\end{tabular}




\begin{tabular}{|c|c|}
\hline $\begin{array}{l}\text { Lian gong } 18 \\
\text { terapias }\end{array}$ & $\begin{array}{l}\text { Prática corporal que promove o fortalecimento e funcionamento } \\
\text { harmonioso do organismo. Trabalha a musculatura, articulações, } \\
\text { tendões e combina técnicas de respiração e relaxamento, favorecendo } \\
\text { a diminuição do tempo de tratamento, aumento da mobilidade } \\
\text { funcional. }\end{array}$ \\
\hline $\begin{array}{l}\text { Medicina e } \\
\text { terapias } \\
\text { antroposóficas }\end{array}$ & $\begin{array}{l}\text { Pratica que amplia a concepção de saúde, buscando visão global do } \\
\text { indivíduo. Usa medicamentos de origem natural e terapias } \\
\text { complementares como massagem rítmica, terapia artística, terapias } \\
\text { ligadas a música e movimentos. }\end{array}$ \\
\hline Meditação & $\begin{array}{l}\text { Prática que facilita o autoconhecimento, autocuidado, } \\
\text { autotransformação e aprimora as inter-relação pessoal, social, } \\
\text { ambiental. Amplia a capacidade de observação, atenção, concentração } \\
\text { e regulação do corpo-mente-emoções. Beneficia o controle da pressão } \\
\text { arterial, frequência cardíaca, redução do estresse, ansiedade, } \\
\text { depressão, melhora a cognição em idosos }\end{array}$ \\
\hline Musicoterapia & $\begin{array}{l}\text { Terapia que utiliza sons, ritmos, melodia, harmonia de forma } \\
\text { terapêutica para a promoção ou recuperação da saúde física, mental, } \\
\text { social e cognitivo do indivíduo ou do grupo com vista na melhora da } \\
\text { qualidade de vida. }\end{array}$ \\
\hline Reike & $\begin{array}{l}\text { Pratica que canaliza frequência energética por meio do toque ou } \\
\text { aproximação das mãos para desbloqueio energético, eliminação de } \\
\text { toxinas e equilíbrio do funcionamento celular de modo a restabelecer } \\
\text { o fluxo de energia vital. }\end{array}$ \\
\hline Shantala & $\begin{array}{l}\text { Terapia realizada por meio de toques e manobras específicas em } \\
\text { bebês, tem como objetivo promover a saúde de modo integral e a } \\
\text { qualidade de vida da criança e família reforçando os vínculos afetivos. } \\
\text { Atua nos sistemas circulatório e linfático, fortalece o sistema } \\
\text { imunológico, atua no sistema respiratório, digestivo e metabólico. }\end{array}$ \\
\hline Tai Chi Chuan & $\begin{array}{l}\text { Terapia realizada por meio da consciência respiratória com } \\
\text { movimentos suaves e naturais. Promove a calma, equilíbrio } \\
\text { emocional, fortalecimento de tendões e articulações, é um exercício } \\
\text { aeróbico leve indicado a pessoas sedentárias e idosas. }\end{array}$ \\
\hline
\end{tabular}




\begin{tabular}{l|l}
\hline $\begin{array}{l}\text { Terapia } \\
\text { comunitária }\end{array}$ & $\begin{array}{l}\text { Prática terapêutica e de produção de saúde em grupo. Constrói laços } \\
\text { sociais, apoio emocional, troca de experiência e prevenção de } \\
\text { integrativa }\end{array}$ \\
& $\begin{array}{l}\text { adoecimento. Funciona como alicerce para a cidadania, rede sociais } \\
\text { solidárias e identidade cultural das comunidades }\end{array}$ \\
\hline
\end{tabular}

Fonte: GERPIS, 2011.

\section{CONCLUSÃO}

O estudo permitiu verificar o aumento progressivo do número de pessoas com 60 anos ou mais no mundo, no Brasil e em Brasília - DF. Dados estatísticos mostram que Brasília tem características diferenciadas do ponto de vista econômico e social das demais unidades da federação, porém tal situação não deixa de evidenciar as necessidades de melhorias efetivas no campo das políticas públicas distritais. E para que seja possível a viabilização da promoção e prevenção em saúde é necessário divulgação ampla e disponibilização de práticas ocupacionais e integrativas que favoreçam a manutenção de uma vida saudável e que essa condição seja uma realidade vivenciada pela pessoa idosa, qualquer que seja a regional de saúde, visto que atualmente a população idosa do DF chega a cerca de $9 \%$ do total da população existente gerando grandes desafios ao setor saúde.

\section{AGRADECIMENTO}

Agradecemos à Faculdade Juscelino Kubitschek, e a todo o corpo docente. Agradecemos também à coordenação do NUSI, que de forma tão atenciosa demonstraram o trabalho realizado pelo núcleo e forneceram dados e materiais relacionados ao tema. Em especial gostaríamos de agradecer aos professores MSc. Paulo Henrique R. Martins e MSc. Danielle Silva Araújo pela colaboração na elaboração do artigo.

\section{REFERÊNCIAS}

BRASIL, Presidência da República, Secretaria de direitos humanos, Secretaria Nacional de Promoção defesa dos direitos humanos. Dados sobre o envelhecimento no Brasil. Brasília (DF); 2013.

BRASIL, Secretaria de Estado de Saúde. Núcleo de Saúde do Idoso. Programas - Saúde do Idoso. Brasília (DF), 1992 
BRASIL, Secretaria de Estado de Saúde. Núcleo de Saúde do Idoso (NUSI). Protocolo de Atenção à Saúde do Idoso. Brasília (DF), 2014.

BRASIL, Secretaria de Estado de Saúde. Gerencia de Informação e Analise de Situação em Saúde/DIVEP / SVS. Resumo da População no Distrito Federal por Regional de Saúde. Brasília (DF), 2014.

BRASIL, Secretaria de Estado de Saúde. Gerencia de Praticas Integrativas em Saúde. Práticas Integrativas em Saúde. Brasília (DF), 2011.

CARNEIRO, L.A.F et al. Envelhecimento populacional e os desafios para o sistema de saúde brasileiro. São Paulo; 2013.

DRUMMOND, A; ALVES, E. D. Perfil socioeconômico e demográfico e a capacidade funcional de idosos atendidos pela Estratégia Saúde da Família de Paranoá Distrito. Rev. bras. Geriatr. Gerontol. V 16 n 4, Rio de Janeiro, 2013.

KANSO, S. Processo de Envelhecimento Populacional: Um Panorama Mundial. Águas de Lindóia, SP; 2013.

KUCHEMANN, B.A. Envelhecimento populacional, cuidado e cidadania: velhos dilemas e novos desafios. Brasília (DF), 2012.

LIMA, C.R.V. Políticas Públicas para idosos: A Realidade das Instituições de Longa Permanência no Distrito Federal. Brasília, 2011.

MIRAgAYA, J. et al. Perfil dos Idosos no Distrito federal, segundo as regiões administrativas. Brasília, 2013.

STIVAL, M.M. et al. Fatores Associados à Qualidade de Vida de Idosos que Frequentam uma unidade de saúde do Distrito Federal. Rev. bras. Geriatr. Gerontol. V 17 n 2, Rio de Janeiro, 2014. 
SAMPIERI, R.H.; COLLADO, C.F.; LUCIO, P. B. Metodologia de Pesquisa. 3.ed. São Paulo: McGraw - Hill, 2006.

VILELA, A.B.A et al. Envelhecimento bem sucedido: Representação de Idosos. Jequié Bahia, 2006. 